\title{
TWINING CHARACTERS AND \\ KOSTANT'S HOMOLOGY FORMULA
}

\author{
SATOSHI NAITO
}

(Received April 17, 2001)

\begin{abstract}
Let $\mathfrak{g}$ be a symmetrizable generalized Kac-Moody algebra and $\mathfrak{n}_{-}$the sum of all its negative root spaces. We obtain a formula for the twining characters of the Lie algebra homology modules of $\mathfrak{n}_{-}$with coefficients in the irreducible highest weight $\mathfrak{g}$-module $L(\Lambda)$ of symmetric, dominant integral highest weight $\Lambda$. This formula gives a new (and convincing) proof of the formula for the twining character of $L(\Lambda)$ above.
\end{abstract}

Introduction. In [FSS] and [FRS], Fuchs, Schweigert, et al. introduced a new type of character-like quantities, called twining characters, corresponding to a Dynkin diagram automorphism for certain highest weight modules over a symmetrizable (generalized) Kac-Moody algebra $\mathfrak{g}$. Moreover, they gave a formula (see Theorem 2.2.1) for the twining character of an irreducible highest weight $\mathfrak{g}$-module $L(\Lambda)$ of symmetric, dominant integral highest weight $\Lambda$.

In this paper, we give a new proof of this result of theirs. In our proof, we use an extension of Kostant's homology formula to generalized Kac-Moody algebras in [N2] to obtain a formula for the twining characters of the Lie algebra homology modules $H_{j}\left(\mathfrak{n}_{-}, L(\Lambda)\right.$ ), $j \geq 0$, of $\mathfrak{n}_{-}$with coefficients in $L(\Lambda)$, where $\mathfrak{n}_{-}$is the sum of all negative root spaces of $\mathfrak{g}$. Then, by an Euler-Poincaré principle, we get the twining character formula for $L(\Lambda)$ of symmetric, dominant integral highest weight $\Lambda$.

This new proof will give us a satisfactory explanation of why we need the subgroup $\tilde{W}$ of the Weyl group $W$ consisting of elements which commute with the Dynkin diagram automorphism.

This paper is organized as follows. In Section 1, we recall the definition of a generalized Kac-Moody algebra and fix our notation. Furthermore, we review an extension of Kostant's homology formula to generalized Kac-Moody algebras in [N2]. In Section 2, following [FSS] and [FRS], we review the definition of a twining character and the twining character formula for $L(\Lambda)$.

Section 3 is the main part of this paper. There we show a formula for the twining characters of the Lie algebra homology modules $H_{j}\left(\mathfrak{n}_{-}, L(\Lambda)\right), j \geq 0$, and then give a new proof of the twining character formula for $L(\Lambda)$.

\section{Preliminaries and notation.}

1.1. Generalized Kac-Moody algebras. Let $I=\{1,2, \ldots, n\}$ be a finite index set, and let $A=\left(a_{i j}\right)_{i, j \in I}$ be an $n \times n$ real matrix satisfying: 
(C1) either $a_{i i}=2$ or $a_{i i} \leq 0$ for all $i \in I$;

(C2) $a_{i j} \leq 0$ if $i \neq j \in I$, and $a_{i j} \in Z$ for $j \neq i$ if $a_{i i}=2$;

(C3) $a_{i j}=0$ if and only if $a_{j i}=0$ for $i, j \in I$.

Such a matrix $A=\left(a_{i j}\right)_{i, j \in I}$ is called a generalized GCM (GGCM). In this paper, following $[\mathrm{K}]$, we define a generalized Kac-Moody algebra (GKM algebra) $\mathfrak{g}$ over $\boldsymbol{C}$ to be the contragredient Lie algebra $\mathfrak{g}(A)$ associated to a GGCM $A=\left(a_{i j}\right)_{i, j \in I}$. Let $\mathfrak{h}$ be the Cartan subalgebra and $e_{i}, f_{i}$ for $i \in I$ the Chevalley generators. Let $\Delta_{+} \subset \mathfrak{h}^{*}:=\operatorname{Hom}_{\boldsymbol{C}}(\mathfrak{h}, \boldsymbol{C})$ be the set of positive roots, $\Delta_{-}=-\Delta_{+}$the set of negative roots, and $\mathfrak{g}_{\alpha}$ the root space of $\mathfrak{g}$ corresponding to a root $\alpha \in \Delta=\Delta_{-} \sqcup \Delta_{+}$. We set

$$
\mathfrak{n}_{ \pm}:=\bigoplus_{\alpha \in \Delta_{ \pm}} \mathfrak{g}_{\alpha}, \quad \mathfrak{b}:=\mathfrak{h} \oplus \mathfrak{n}_{+} .
$$

We denote by $\Pi=\left\{\alpha_{i} \mid i \in I\right\}$ the set of simple roots, and by $\Pi^{\vee}=\left\{h_{i} \mid i \in I\right\}$ the set of simple coroots. We set $I^{\mathrm{re}}:=\left\{i \in I \mid a_{i i}=2\right\}, I^{\mathrm{im}}:=\left\{i \in I \mid a_{i i} \leq 0\right\}$, and call $\Pi^{\mathrm{re}}:=\left\{\alpha_{i} \in \Pi \mid i \in I^{\mathrm{re}}\right\}$ the set of real simple roots, $\Pi^{\mathrm{im}}:=\left\{\alpha_{i} \in \Pi \mid i \in I^{\mathrm{im}}\right\}$ the set of imaginary simple roots. Note that $\mathfrak{g}_{\alpha_{i}}=\boldsymbol{C} e_{i}, \mathfrak{g}_{-\alpha_{i}}=\boldsymbol{C} f_{i}$ for all $i \in I$.

The Weyl group $W$ of the GKM algebra $\mathfrak{g}$ is defined by

$$
W:=\left\langle r_{i} \mid i \in I^{\mathrm{re}}\right\rangle \subset G L\left(\mathfrak{h}^{*}\right),
$$

where $r_{i} \in G L\left(\mathfrak{h}^{*}\right)$ for $i \in I^{\mathrm{re}}$ is the simple reflection of $\mathfrak{h}^{*}$. The length function of the Coxeter system $\left(W,\left\{r_{i} \mid i \in I^{\mathrm{re}}\right\}\right)$ is denoted by

$$
\ell: W \rightarrow Z \text {. }
$$

Throughout this paper, we assume that a GGCM $A=\left(a_{i j}\right)_{i, j \in I}$ is symmetrizable, i.e., that there exist a diagonal matrix $D=\operatorname{diag}\left(\varepsilon_{1}, \ldots, \varepsilon_{n}\right)$ with $\varepsilon_{i}>0$ for all $i \in I$ and a symmetric matrix $B=\left(b_{i j}\right)_{i, j \in I}$ such that $A=D B$. Hence there exists a nondegenerate, symmetric, invariant bilinear form $(\cdot \mid \cdot)$ on $\mathfrak{g}=\mathfrak{g}(A)$. The restriction of this bilinear form $(\cdot \mid \cdot)$ to $\mathfrak{h}$ is again nondegenerate, so that it induces a nondegenerate, symmetric, $W$-invariant bilinear form on $\mathfrak{h}^{*}$, which is also denoted by $(\cdot \mid \cdot)$.

1.2. Kostant's homology formula. For $\lambda \in \mathfrak{h}^{*}$, let

$$
M(\lambda):=U(\mathfrak{g}) \otimes_{U(\mathfrak{b})} \boldsymbol{C}(\lambda)
$$

be the Verma module of highest weight $\lambda$ over $\mathfrak{g}$, where $U(\mathfrak{a})$ denotes the universal enveloping algebra of a Lie algebra $\mathfrak{a}$ and $\boldsymbol{C}(\lambda)$ is the one-dimensional (irreducible) $\mathfrak{h}$-module of weight $\lambda$ on which $\mathfrak{n}_{+}$acts trivially. We then define a $\mathfrak{g}$-module $L(\lambda)$ to be the unique irreducible quotient of $M(\lambda)$, that is,

$$
L(\lambda):=M(\lambda) / J(\lambda),
$$

where $J(\lambda)$ is the unique maximal proper submodule of $M(\lambda)$.

Let

$$
P_{+}:=\left\{\Lambda \in \mathfrak{h}^{*} \mid \Lambda\left(h_{i}\right) \geq 0 \text { for all } i \in I \text {, and } \Lambda\left(h_{i}\right) \in \boldsymbol{Z} \text { if } a_{i i}=2\right\}
$$


be the set of dominant integral weights. We denote by $H_{j}\left(\mathfrak{n}_{-}, L(\Lambda)\right), j \geq 0$, the Lie algebra homology modules of $\mathfrak{n}_{-}$with coefficients in $L(\Lambda)$ for $\Lambda \in P_{+}$. Recall from [GL] that, for $j \geq 0$, the $H_{j}\left(\mathfrak{n}_{-}, L(\Lambda)\right)$ is defined to be the $j$-th homology of the chain complex $\left\{C_{j}\left(\mathfrak{n}_{-}, L(\Lambda)\right), d_{j}\right\}_{j \geq 0}$ with $C_{j}\left(\mathfrak{n}_{-}, L(\Lambda)\right):=\left(\bigwedge^{j} \mathfrak{n}_{-}\right) \otimes_{C} L(\Lambda)$, where $\bigwedge^{j} \mathfrak{n}_{-}$denotes the $j$-th exterior power of $\mathfrak{n}_{-}$. Note that the boundary operator $d_{j}: C_{j}\left(\mathfrak{n}_{-}, L(\Lambda)\right) \rightarrow$ $C_{j-1}\left(\mathfrak{n}_{-}, L(\Lambda)\right)$ commutes with the action of $\mathfrak{h}$, and hence $H_{j}\left(\mathfrak{n}_{-}, L(\Lambda)\right)$ is an $\mathfrak{h}$-module in the usual way.

In order to state an extension of Kostant's homology formula to GKM algebras, we introduce some notation. Let $i \neq j \in I^{\mathrm{im}}$ and $\Lambda \in P_{+}$. Two distinct imaginary simple roots $\alpha_{i}$ and $\alpha_{j}$ are said to be pairwise perpendicular if $a_{i j}=0=a_{j i}$, and an imaginary simple root $\alpha_{i}$ is said to be perpendicular to $\Lambda$ if $\Lambda\left(h_{i}\right)=0$. We denote by $\mathcal{S}(\Lambda)$ the set of sums of distinct, pairwise perpendicular, imaginary simple roots perpendicular to $\Lambda$. In addition, for an element $\beta=\sum_{i \in I^{\text {im }}} k_{i} \alpha_{i} \in \mathcal{S}(\Lambda)$, we set ht $(\beta):=\sum_{i \in I^{\text {im }}} k_{i} \in \boldsymbol{Z}_{\geq 0}$ (note that $k_{i}=0,1$ for all $i \in I^{\mathrm{im}}$ by the definition of $\mathcal{S}(\Lambda)$ ). Now we take and fix an element $\rho \in \mathfrak{h}^{*}$ (called a Weyl vector) such that $\rho\left(h_{i}\right)=(1 / 2) \cdot a_{i i}$ for all $i \in I$. For $(w, \beta) \in W \times \mathcal{S}(\Lambda)$, we set

$$
(w, \beta) \circ \Lambda:=w(\Lambda+\rho-\beta)-\rho .
$$

We know from [N2, Propositions 3.2, 3.3, and Theorem 5.3] the following theorem.

THEOREM 1.2.1. Let $\Lambda \in P_{+}$and $j \in Z_{\geq 0}$.

(1) We have the following isomorphism of $\mathfrak{h}$-modules:

$$
H_{j}\left(\mathfrak{n}_{-}, L(\Lambda)\right) \cong \bigoplus_{\substack{(w, \beta) \in W \times \mathcal{S}(\Lambda) \\ \ell(w)+\mathrm{ht}(\beta)=j}} \boldsymbol{C}((w, \beta) \circ \Lambda)
$$

Here the sum above is a direct sum of inequivalent irreducible $\mathfrak{h}$-modules, i.e., the weights $(w, \beta) \circ \Lambda$ for $(w, \beta) \in W \times \mathcal{S}(\Lambda)$ with $\ell(w)+\mathrm{ht}(\beta)=j$ are all distinct.

(2) If we set $\mu:=(w, \beta) \circ \Lambda$ for $(w, \beta) \in W \times \mathcal{S}(\Lambda)$ with $\ell(w)+\operatorname{ht}(\beta)=j$, then the multiplicities of $\mu\left(=\right.$ the dimensions of the $\mu$-weight space) in the $\mathfrak{h}$-modules $\left(\bigwedge^{*} \mathfrak{n}_{-}\right) \otimes_{\boldsymbol{C}}$ $L(\Lambda)$ and $\left(\bigwedge^{j} \mathfrak{n}_{-}\right) \otimes_{C} L(\Lambda)$ are both equal to one.

Here we recall from the proof of [N2, Proposition 3.3] the construction of a nonzero weight vector $v_{(w, \beta)} \in\left(\bigwedge^{j} \mathfrak{n}_{-}\right) \otimes_{C} L(\Lambda)$ of weight $\mu=(w, \beta) \circ \Lambda$ in part (2) of Theorem 1.2.1. First, we note that $w(\rho)-\rho=-\sum_{\alpha \in \Delta_{w}} \alpha$ and that the number of elements of the set $\Delta_{w}$ equals $\ell(w)$, where $\Delta_{w}:=\left\{\alpha \in \Delta_{+} \mid w^{-1}(\alpha) \in \Delta_{-}\right\}$. Second, we write $\beta$ in the form $\beta=\sum_{k=1}^{m} \alpha_{i_{k}}$, where $m=\mathrm{ht}(\beta), \alpha_{i_{k}} \in \Pi^{\mathrm{im}}$, and $i_{r} \neq i_{t}$ for $1 \leq r \neq t \leq m$. Now we take nonzero root vectors $F_{k} \in \mathfrak{g}_{-w\left(\alpha_{i_{k}}\right)}$ for $1 \leq k \leq m, F_{\alpha} \in \mathfrak{g}_{-\alpha}$ for $\alpha \in \Delta_{w}$, and a nonzero weight vector $v_{w(\Lambda)} \in L(\Lambda)_{w(\Lambda)}$ of weight $w(\Lambda)$. Then we set

$$
v_{(w, \beta)}:=\left(F_{1} \wedge \cdots \wedge F_{m}\right) \wedge\left(\bigwedge_{\alpha \in \Delta_{w}} F_{\alpha}\right) \otimes v_{w(\Lambda)} \in\left(\bigwedge^{j} \mathfrak{n}_{-}\right) \otimes_{C} L(\Lambda) .
$$


We see that the vector $v_{(w, \beta)} \in\left(\bigwedge^{j} \mathfrak{n}_{-}\right) \otimes_{\boldsymbol{C}} L(\Lambda)$ is nonzero, and of weight $\mu=(w, \beta) \circ \Lambda$, since

$$
\begin{aligned}
\mu & =w(\Lambda+\rho-\beta)-\rho \\
& =-w(\beta)+(w(\rho)-\rho)+w(\Lambda) \\
& =\sum_{k=1}^{m}\left(-w\left(\alpha_{i_{k}}\right)\right)+\sum_{\alpha \in \Delta_{w}}(-\alpha)+w(\Lambda) .
\end{aligned}
$$

We deduce from Theorem 1.2.1 that the image $\bar{v}_{(w, \beta)}$ of the vector $v_{(w, \beta)} \in\left(\bigwedge^{j} \mathfrak{n}_{-}\right) \otimes_{\boldsymbol{C}}$ $L(\Lambda)$ of weight $\mu$ by the natural quotient map ${ }^{-}:\left(\bigwedge^{j} \mathfrak{n}_{-}\right) \otimes_{C} L(\Lambda) \rightarrow H_{j}\left(\mathfrak{n}_{-}, L(\Lambda)\right)$ is nonzero, and hence that the $\mu$-weight space $\left(H_{j}\left(\mathfrak{n}_{-}, L(\Lambda)\right)\right)_{\mu}$ of $H_{j}\left(\mathfrak{n}_{-}, L(\Lambda)\right)$ is spanned by the vector $\bar{v}_{(w, \beta)}$ :

$$
\left(H_{j}\left(\mathfrak{n}_{-}, L(\Lambda)\right)\right)_{\mu}=\boldsymbol{C} \bar{v}_{(w, \beta)} .
$$

\section{Twining character formula for $L(\Lambda)$.}

2.1. Twining characters. We recall the definition of the twining character of a certain highest weight module, following [FRS] and [FSS] (see also [N4]).

Let $A=\left(a_{i j}\right)_{i, j \in I}$ be a symmetrizable GGCM indexed by a finite set $I$. A bijection $\omega: I \rightarrow I$ such that

$$
a_{\omega(i), \omega(j)}=a_{i j} \quad \text { for all } i, j \in I
$$

is called a (Dynkin) diagram automorphism, since such an $\omega$ induces an automorphism of the Dynkin diagram of the GGCM $A=\left(a_{i j}\right)_{i, j \in I}$ as a graph. Let $N$ be the order of $\omega: I \rightarrow I$, and $N_{i}$ the number of elements of the $\omega$-orbit of $i \in I$ in $I$. We may (and will henceforth) assume that $\varepsilon_{\omega(i)}=\varepsilon_{i}$ for all $i \in I$ in the decomposition $A=D B$ with $D=\operatorname{diag}\left(\varepsilon_{1}, \ldots, \varepsilon_{n}\right)$ (see $[\mathrm{N} 4, \S 3.1]$ ).

The diagram automorphism $\omega: I \rightarrow I$ can be extended (cf. [FSS, §3.2] and [K, §2.2]) to an automorphism $\omega$ of order $N$ of the GKM algebra $\mathfrak{g}=\mathfrak{g}(A)$ associated to the GGCM $A=\left(a_{i j}\right)_{i, j \in I}$ so that

$$
\begin{cases}\omega\left(e_{i}\right):=e_{\omega(i)} & \text { for } i \in I, \\ \omega\left(f_{i}\right):=f_{\omega(i)} & \text { for } i \in I, \\ \omega\left(h_{i}\right):=h_{\omega(i)} & \text { for } i \in I, \\ \omega(\mathfrak{h}):=\mathfrak{h}, & \text { for } x, y \in \mathfrak{g} .\end{cases}
$$

Notice that the $\omega: \mathfrak{g} \rightarrow \mathfrak{g}$ extends to a unique algebra automorphism $\omega: U(\mathfrak{g}) \rightarrow U(\mathfrak{g})$ by

$$
\omega\left(x_{1} \cdots x_{k}\right)=\omega\left(x_{1}\right) \cdots \omega\left(x_{k}\right) \text { for } x_{1}, \ldots, x_{k} \in \mathfrak{g} .
$$

We call these two automorphisms $\omega$ also diagram automorphisms by abuse of notation. 
The restriction of the diagram automorphism $\omega: \mathfrak{g} \rightarrow \mathfrak{g}$ to the Cartan subalgebra $\mathfrak{h}$ induces a transposed map $\omega^{*}: \mathfrak{h}^{*} \rightarrow \mathfrak{h}^{*}$ by

$$
\omega^{*}(\lambda)(h):=\lambda(\omega(h)) \quad \text { for } \lambda \in \mathfrak{h}^{*}, h \in \mathfrak{h} .
$$

We set

$$
\left(\mathfrak{h}^{*}\right)^{0}:=\left\{\lambda \in \mathfrak{h}^{*} \mid \omega^{*}(\lambda)=\lambda\right\},
$$

and call an element of $\left(\mathfrak{h}^{*}\right)^{0}$ a symmetric weight. Note that we may (and will henceforth) assume that the Weyl vector $\rho$ is a symmetric weight, i.e.,

$$
\omega^{*}(\rho)=\rho .
$$

Let $\lambda \in\left(\mathfrak{h}^{*}\right)^{0}$ be a symmetric weight, and let $V(\lambda)$ be either the Verma module $M(\lambda)$ or the irreducible highest weight module $L(\lambda)$ of highest weight $\lambda$. Then there exists a unique linear automorphism $\tau_{\omega}: V(\lambda) \rightarrow V(\lambda)$ such that

$$
\tau_{\omega}(x v)=\omega^{-1}(x) \tau_{\omega}(v) \quad \text { for } x \in \mathfrak{g}, v \in V(\lambda),
$$

and

$$
\tau_{\omega}(v)=v \quad \text { for } v \in V(\lambda)_{\lambda},
$$

where $V(\lambda)_{\lambda}$ is the (one-dimensional) highest weight space of $V(\lambda)$.

Remark 2.1.1. Because $M(\lambda)=U(\mathfrak{g}) \otimes_{U(\mathfrak{b})} \boldsymbol{C}(\lambda)$ by definition, we can take the linear automorphism $\omega^{-1} \otimes \mathrm{id}: U(\mathfrak{g}) \otimes_{U(\mathfrak{b})} \boldsymbol{C}(\lambda) \rightarrow U(\mathfrak{g}) \otimes_{U(\mathfrak{b})} \boldsymbol{C}(\lambda)$ for $\tau_{\omega}: M(\lambda) \rightarrow M(\lambda)$ above. Moreover, since this map $\omega^{-1} \otimes$ id $: M(\lambda) \rightarrow M(\lambda)$ stabilizes the unique maximal proper submodule $J(\lambda)$ of $M(\lambda)$, we can take for $\tau_{\omega}: L(\lambda) \rightarrow L(\lambda)$ above the linear map $M(\lambda) / J(\lambda) \rightarrow M(\lambda) / J(\lambda)$ induced from $\omega^{-1} \otimes \mathrm{id}: M(\lambda) \rightarrow M(\lambda)$.

REMARK 2.1.2. Let $V$ be an $\mathfrak{h}$-module admitting a weight space decomposition

$$
V=\bigoplus_{\chi \in \mathfrak{h}^{*}} V_{\chi}
$$

with finite-dimensional weight spaces $V_{\chi}$, and let $f: V \rightarrow V$ be a linear map such that $f(h v)=\omega^{-1}(h) f(v)$ for $h \in \mathfrak{h}, v \in V$. Then it follows that

$$
f\left(V_{\chi}\right) \subset V_{\omega^{*}}(\chi)
$$

for all $\chi \in \mathfrak{h}^{*}$. Thus, we define a formal sum:

$$
\operatorname{Tr}_{V} f \exp :=\sum_{\chi \in\left(\mathfrak{h}^{*}\right)^{0}} \operatorname{Tr}\left(\left.f\right|_{V_{\chi}}\right) e(\chi),
$$

where $\operatorname{Tr}\left(\left.f\right|_{V_{\chi}}\right)$ for $\chi \in\left(\mathfrak{h}^{*}\right)^{0}$ denotes the trace of the restriction of $f$ to the $\chi$-weight space $V_{\chi}$ of $V$. 
Let $\lambda \in\left(\mathfrak{h}^{*}\right)^{0}$ be a symmetric weight. The twining character $\operatorname{ch}^{\omega}(V(\lambda))$ of $V(\lambda)$ $(=M(\lambda), L(\lambda))$ is then defined to be the formal sum

$$
\operatorname{ch}^{\omega}(V(\lambda)):=\operatorname{Tr}_{V(\lambda)} \tau_{\omega} \exp =\sum_{\chi \in\left(\mathfrak{h}^{*}\right)^{0}} \operatorname{Tr}\left(\left.\tau_{\omega}\right|_{V(\lambda)_{\chi}}\right) e(\chi) .
$$

2.2. Twining character formula for $L(\Lambda)$. We review the twining character formula for $L(\Lambda)$ of symmetric, dominant integral highest weight $\Lambda$, which is the main result of [FSS] and [FRS].

We choose a set of representatives $\hat{I}$ of the $\omega$-orbits in $I$, and then introduce the following subset of $\hat{I}$ :

$$
\breve{I}:=\left\{i \in \hat{I} \mid \sum_{k=0}^{N_{i}-1} a_{i, \omega^{k}(i)}=1,2\right\} .
$$

We define the following subgroup of the Weyl group $W$ :

$$
\tilde{W}:=\left\{w \in W \mid \omega^{*} w=w \omega^{*}\right\} .
$$

We know from [FRS, Proposition 3.3] that the group $\tilde{W}$ is a Coxeter group with the canonical generator system $\left\{w_{i} \mid i \in \breve{I}\right\}$, where for $i \in \breve{I}$,

$$
w_{i}:= \begin{cases}\prod_{k=0}^{N_{i} / 2-1}\left(r_{\omega^{k}(i)} r_{\omega^{k+N_{i} / 2}(i)} r_{\omega^{k}(i)}\right) & \text { if } \sum_{k=0}^{N_{i}-1} a_{i, \omega^{k}(i)}=1, \\ \prod_{k=0}^{N_{i}-1} r_{\omega^{k}(i)} & \text { if } \sum_{k=0}^{N_{i}-1} a_{i, \omega^{k}(i)}=2 .\end{cases}
$$

Here we note that if $\sum_{k=0}^{N_{i}-1} a_{i, \omega^{k}(i)}=1$, then $N_{i}$ is an even integer. We denote the length function of the Coxeter system $\left(\tilde{W},\left\{w_{i} \mid i \in \breve{I}\right\}\right)$ by

$$
\hat{\ell}: \tilde{W} \rightarrow \boldsymbol{Z} \text {. }
$$

We also recall from [FRS, Equation (1) on page 529] that for a symmetric weight $\lambda \in\left(\mathfrak{h}^{*}\right)^{0}$ and $i \in \breve{I}$,

$$
w_{i}(\lambda)=\lambda-\frac{2 s_{i}\left(\lambda \mid \alpha_{i}\right)}{\left(\alpha_{i} \mid \alpha_{i}\right)} \sum_{k=0}^{N_{i}-1} \alpha_{\omega^{k}(i)}
$$

where $s_{i}:=2 / \sum_{k=0}^{N_{i}-1} a_{i, \omega^{k}(i)}$.

If $\Lambda \in P_{+} \cap\left(\mathfrak{h}^{*}\right)^{0}$ is a symmetric, dominant integral weight, then each element $\beta \in$ $\mathcal{S}(\Lambda) \cap\left(\mathfrak{h}^{*}\right)^{0}$ can be written in the form $\beta=\sum_{i \in \hat{I}} k_{i} \beta_{i}$, where $\beta_{i}:=\sum_{k=0}^{N_{i}-1} \alpha_{\omega^{k}(i)} \in\left(\mathfrak{h}^{*}\right)^{0}$ and $k_{i}=0,1$ for $i \in \hat{I}$. For such a $\beta \in \mathcal{S}(\Lambda) \cap\left(\mathfrak{h}^{*}\right)^{0}$, we set

$$
\widehat{\operatorname{ht}}(\beta):=\sum_{i \in \hat{I}} k_{i} \text {. }
$$


Let $\Lambda \in P_{+} \cap\left(\mathfrak{h}^{*}\right)^{0}$ be a symmetric, dominant integral weight. We know from [FRS, Proposition 3.5] that for every $w \in \tilde{W}$,

$$
w\left(\operatorname{ch}^{\omega}(L(\Lambda))\right)=\operatorname{ch}^{\omega}(L(\Lambda)),
$$

since the $\mathfrak{g}$-module $L(\Lambda)$ is integrable. Moreover, we have

THEOREM 2.2.1 ([FRS, Theorem 3.1]). Let $\Lambda \in P_{+} \cap\left(\mathfrak{h}^{*}\right)^{0}$ be a symmetric, dominant integral weight. Then

$$
\operatorname{ch}^{\omega}(L(\Lambda))=\frac{\sum_{\substack{w \in \tilde{W} \\ \beta \in \mathcal{S}(\Lambda) \cap\left(\mathfrak{h}^{*}\right)^{0}}}(-1)^{\hat{\ell}(w)+\widehat{\mathrm{ht}}(\beta)} e((w, \beta) \circ \Lambda)}{\sum_{\substack{w \in \tilde{W} \\ \beta \in \mathcal{S}(0) \cap\left(\mathfrak{h}^{*}\right)^{0}}}(-1)^{\hat{\ell}(w)+\widehat{\mathrm{ht}}(\beta)} e((w, \beta) \circ 0)} .
$$

\section{Twining character formula for $H_{j}\left(\mathfrak{n}_{-}, L(\Lambda)\right)$.}

3.1. Some lemmas. Since the inverse $\omega^{-1}: \mathfrak{g} \rightarrow \mathfrak{g}$ of the diagram automorphism $\omega: \mathfrak{g} \rightarrow \mathfrak{g}$ stabilizes $\mathfrak{n}_{-}$, i.e., $\omega^{-1}\left(\mathfrak{n}_{-}\right)=\mathfrak{n}_{-}$, it induces an algebra automorphism

$$
\bigwedge^{*} \omega^{-1}: \bigwedge^{*} \mathfrak{n}_{-} \rightarrow \bigwedge^{*} \mathfrak{n}_{-}
$$

of the exterior algebra $\Lambda^{*} \mathfrak{n}_{-}$of $\mathfrak{n}_{-}$. The restriction of the $\Lambda^{*} \omega^{-1}: \Lambda^{*} \mathfrak{n}_{-} \rightarrow \Lambda^{*} \mathfrak{n}_{-}$to each homogeneous subspace $\wedge^{j} \mathfrak{n}_{-}$for $j \geq 0$ is denoted by

$$
\bigwedge^{j} \omega^{-1}: \bigwedge^{j} \mathfrak{n}_{-} \rightarrow \bigwedge^{j} \mathfrak{n}_{-}
$$

Let $\Lambda \in P_{+} \cap\left(\mathfrak{h}^{*}\right)^{0}$ be a symmetric, dominant integral weight, and let $\tau_{\omega}: L(\Lambda) \rightarrow$ $L(\Lambda)$ be the linear automorphism in Section 2.1. We define a linear automorphism

$$
\Phi:=\left(\bigwedge^{*} \omega^{-1}\right) \otimes \tau_{\omega}:\left(\bigwedge^{*} \mathfrak{n}_{-}\right) \otimes_{\boldsymbol{C}} L(\Lambda) \rightarrow\left(\bigwedge^{*} \mathfrak{n}_{-}\right) \otimes_{\boldsymbol{C}} L(\Lambda),
$$

and for $j \geq 0$, we define a linear automorphism

$$
\Phi_{j}:=\left(\bigwedge^{j} \omega^{-1}\right) \otimes \tau_{\omega}:\left(\bigwedge^{j} \mathfrak{n}_{-}\right) \otimes_{\boldsymbol{C}} L(\Lambda) \rightarrow\left(\bigwedge^{j} \mathfrak{n}_{-}\right) \otimes_{\boldsymbol{C}} L(\Lambda) .
$$

REMARK 3.1.1. Let $j \geq 0$. It is easily seen that

$$
\Phi_{j}(h v)=\omega^{-1}(h) \Phi_{j}(v)
$$

for $h \in \mathfrak{h}$ and $v \in\left(\bigwedge^{j} \mathfrak{n}_{-}\right) \otimes_{\boldsymbol{C}} L(\Lambda)$. It also follows that for $h \in \mathfrak{h}$ and $v \in\left(\bigwedge^{*} \mathfrak{n}_{-}\right) \otimes_{\boldsymbol{C}} L(\Lambda)$,

$$
\Phi(h v)=\omega^{-1}(h) \Phi(v) .
$$

The follwing lemma immediately follows from the definitions of $d_{j}$ and $\Phi_{j}, j \geq 0$. 
LEMMA 3.1.2. We have the following commutative diagram for each $j \geq 0$ :

$$
\begin{array}{ccc}
\left(\bigwedge^{j} \mathfrak{n}_{-}\right) \otimes_{\boldsymbol{C}} L(\Lambda) & \stackrel{\Phi_{j}}{\longrightarrow} & \left(\bigwedge^{j} \mathfrak{n}_{-}\right) \otimes_{\boldsymbol{C}} L(\Lambda) \\
d_{j} \downarrow & \downarrow d_{j} \\
\left(\bigwedge^{j-1} \mathfrak{n}_{-}\right) \otimes_{\boldsymbol{C}} L(\Lambda) \underset{\Phi_{j-1}}{\longrightarrow} & \left(\bigwedge^{j-1} \mathfrak{n}_{-}\right) \otimes_{\boldsymbol{C}} L(\Lambda),
\end{array}
$$

where $d_{j}:\left(\bigwedge^{j} \mathfrak{n}_{-}\right) \otimes_{C} L(\Lambda) \rightarrow\left(\bigwedge^{j-1} \mathfrak{n}_{-}\right) \otimes_{C} L(\Lambda)$ is the boundary operator in Section 1.2 .

By Lemma 3.1.2, the linear automorphism $\Phi_{j}:\left(\bigwedge^{j} \mathfrak{n}_{-}\right) \otimes_{C} L(\Lambda) \rightarrow\left(\bigwedge^{j} \mathfrak{n}_{-}\right) \otimes_{C} L(\Lambda)$ induces in the usual way a linear automorphism

$$
\bar{\Phi}_{j}: H_{j}\left(\mathfrak{n}_{-}, L(\Lambda)\right) \rightarrow H_{j}\left(\mathfrak{n}_{-}, L(\Lambda)\right)
$$

for $j \geq 0$. Notice that for $j \geq 0$ and $h \in \mathfrak{h}, v \in H_{j}\left(\mathfrak{n}_{-}, L(\Lambda)\right)$,

$$
\bar{\Phi}_{j}(h v)=\omega^{-1}(h) \bar{\Phi}_{j}(v)
$$

by Remark 3.1.1.

Now we state an easy but useful lemma, which will be often used later. Let $V$ and $V^{\prime}$ be $\mathfrak{h}$-modules which decompose into a direct sum of finite-dimensional weight spaces:

$$
V=\bigoplus_{\chi \in \mathfrak{h}^{*}} V_{\chi} \quad \text { and } \quad V^{\prime}=\bigoplus_{\chi \in \mathfrak{h}^{*}} V_{\chi}^{\prime} .
$$

We further assume that there exist linear automorphisms $\tau_{\omega}: V \rightarrow V$ and $\tau_{\omega}^{\prime}: V^{\prime} \rightarrow V^{\prime}$ such that for $h \in \mathfrak{h}$,

$$
\tau_{\omega}(h v)=\omega^{-1}(h) \tau_{\omega}(v) \text { for } v \in V \text {, and } \tau_{\omega}^{\prime}(h v)=\omega^{-1}(h) \tau_{\omega}^{\prime}(v) \text { for } v \in V^{\prime} .
$$

Then the linear automorphism

$$
\tau_{\omega} \otimes \tau_{\omega}^{\prime}: V \otimes_{C} V^{\prime} \rightarrow V \otimes_{C} V^{\prime}
$$

obviously satisfies

$$
\left(\tau_{\omega} \otimes \tau_{\omega}^{\prime}\right)(h v)=\omega^{-1}(h)\left(\tau_{\omega} \otimes \tau_{\omega}^{\prime}\right)(v)
$$

for $h \in \mathfrak{h}$ and $v \in V \otimes_{\boldsymbol{C}} V^{\prime}$. We can easily show

LEMMA 3.1.3. In the notation above, we have

$$
\operatorname{Tr}_{V \otimes V^{\prime}}\left(\tau_{\omega} \otimes \tau_{\omega}^{\prime}\right) \exp =\left(\operatorname{Tr}_{V} \tau_{\omega} \exp \right) \cdot\left(\operatorname{Tr}_{V^{\prime}} \tau_{\omega}^{\prime} \exp \right) .
$$

3.2. Main result. We define the twining character $\operatorname{ch}^{\omega}\left(H_{j}\left(\mathfrak{n}_{-}, L(\Lambda)\right)\right)$ of the Lie algebra homology module $H_{j}\left(\mathfrak{n}_{-}, L(\Lambda)\right)$ for each $j \geq 0$ by

$$
\operatorname{ch}^{\omega}\left(H_{j}\left(\mathfrak{n}_{-}, L(\Lambda)\right)\right):=\operatorname{Tr}_{H_{j}\left(\mathfrak{n}_{-}, L(\Lambda)\right)} \bar{\Phi}_{j} \exp ,
$$

where $\bar{\Phi}_{j}: H_{j}\left(\mathfrak{n}_{-}, L(\Lambda)\right) \rightarrow H_{j}\left(\mathfrak{n}_{-}, L(\Lambda)\right)$ is as in Section 3.1 . 
Proposition 3.2.1. Let $\Lambda \in P_{+} \cap\left(\mathfrak{h}^{*}\right)^{0}$ be a symmetric, dominant integral weight, and let $j \geq 0$. Then

$$
\operatorname{ch}^{\omega}\left(H_{j}\left(\mathfrak{n}_{-}, L(\Lambda)\right)\right)=\sum_{\substack{w \in \tilde{W} \\ \beta \in \mathcal{S}(\Lambda) \cap\left(\mathfrak{h}^{*}\right)^{0} \\ \ell(w)+h \mathfrak{h t}(\beta)=j}} c_{(w, \beta)} e((w, \beta) \circ \Lambda),
$$

where the scalar $c_{(w, \beta)} \in \boldsymbol{C}$ is defined by

$$
c_{(w, \beta)}:=\operatorname{Tr}\left(\left.\bar{\Phi}_{j}\right|_{\left(H_{j}\left(\mathfrak{n}_{-}, L(\Lambda)\right)\right)_{(w, \beta) \circ \Lambda}}\right) .
$$

Proof. Let $w \in W$ and $\beta \in \mathcal{S}(\Lambda)$ with $\ell(w)+\operatorname{ht}(\beta)=j$. Set $\mu:=(w, \beta) \circ \Lambda$. Let us show that $\omega^{*}(\mu)=\mu$ if and only if $w \in \tilde{W}$ and $\beta \in\left(\mathfrak{h}^{*}\right)^{0}$. Because $\omega^{*} r_{i}\left(\omega^{*}\right)^{-1}=r_{\omega^{-1}(i)}$ for $i \in I^{\text {re }}$, we see that $\omega^{*} w\left(\omega^{*}\right)^{-1} \in W$. If we set $w^{\prime}:=\omega^{*} w\left(\omega^{*}\right)^{-1} \in W$, then we have

$$
\begin{aligned}
\omega^{*}(\mu) & =\omega^{*}(w(\Lambda+\rho-\beta)-\rho) \\
& =\omega^{*}(w(\Lambda+\rho-\beta))-\omega^{*}(\rho) \\
& =\omega^{*} w(\Lambda+\rho-\beta)-\rho \\
& =w^{\prime} \omega^{*}(\Lambda+\rho-\beta)-\rho \\
& =w^{\prime}\left(\omega^{*}(\Lambda+\rho-\beta)\right)-\rho \\
& =w^{\prime}\left(\Lambda+\rho-\omega^{*}(\beta)\right)-\rho,
\end{aligned}
$$

since $\omega^{*}(\Lambda)=\Lambda$ and $\omega^{*}(\rho)=\rho$. Now assume that $\omega^{*}(\mu)=\mu$, i.e., that $w^{\prime}(\Lambda+\rho-$ $\left.\omega^{*}(\beta)\right)=w(\Lambda+\rho-\beta)$. Then, since $(\Lambda+\rho-\beta)\left(h_{i}\right) \geq 1$ and $\left(\Lambda+\rho-\omega^{*}(\beta)\right)\left(h_{i}\right) \geq 1$ for all $i \in I^{\text {re }}$, we deduce that $\Lambda+\rho-\omega^{*}(\beta)=\Lambda+\rho-\beta$ (i.e., $\omega^{*}(\beta)=\beta$ ) and that $w^{\prime}=w$ (i.e., $\omega^{*} w\left(\omega^{*}\right)^{-1}=w$ ) by the proof of [K, Proposition 3.12 a) and b)]. Conversely, $\omega^{*} w\left(\omega^{*}\right)^{-1}=$ $w$ and $\omega^{*}(\beta)=\beta$ immediately imply that $\omega^{*}(\mu)=\mu$. Therefore, the proposition follows directly from Theorem 1.2.1 and the definition (3.2.1) of $\operatorname{ch}^{\omega}\left(H_{j}\left(\mathfrak{n}_{-}, L(\Lambda)\right)\right)$.

We see from the comments just below Theorem 1.2.1 that for $w \in \tilde{W}$ and $\beta \in \mathcal{S}(\Lambda) \cap$ $\left(\mathfrak{h}^{*}\right)^{0}$ with $\ell(w)+\mathrm{ht}(\beta)=j$,

$$
\begin{aligned}
c_{(w, \beta)} & =\operatorname{Tr}\left(\left.\bar{\Phi}_{j}\right|_{\left(H_{j}\left(\mathfrak{n}_{-}, L(\Lambda)\right)\right)_{(w, \beta) \perp \Lambda}}\right) \\
& =\operatorname{Tr}\left(\left.\bar{\Phi}_{j}\right|_{\boldsymbol{C}} \bar{v}_{(w, \beta)}\right) \\
& =\operatorname{Tr}\left(\left.\Phi_{j}\right|_{\boldsymbol{C}} v_{(w, \beta)}\right) \\
& =\operatorname{Tr}\left(\left.\Phi_{j}\right|_{\left(\left(\bigwedge^{j} \mathfrak{n}_{-}\right) \otimes_{C} L(\Lambda)\right)_{(w, \beta) \perp \Lambda}}\right) .
\end{aligned}
$$

To determine the scalar $c_{(w, \beta)} \in \boldsymbol{C}$, we define the twining character $\operatorname{ch}^{\omega}\left(\left(\bigwedge^{*} \mathfrak{n}_{-}\right) \otimes_{\boldsymbol{C}}\right.$ $L(\Lambda))$ of $\left(\bigwedge^{*} \mathfrak{n}_{-}\right) \otimes_{C} L(\Lambda)$ by

$$
\operatorname{ch}^{\omega}\left(\left(\bigwedge^{*} \mathfrak{n}_{-}\right) \otimes_{C} L(\Lambda)\right):=\operatorname{Tr}_{\left(\bigwedge^{*} \mathfrak{n}_{-}\right) \otimes_{C} L(\Lambda)} \Phi \exp ,
$$

where $\Phi=\left(\bigwedge^{*} \omega^{-1}\right) \otimes \tau_{\omega}$ is as in Section 3.1. The following is our key proposition. 
Proposition 3.2.2. Let $\Lambda \in P_{+} \cap\left(\mathfrak{h}^{*}\right)^{0}$ be a symmetric, dominant integral weight. For every $w \in \tilde{W}$, we have

$w\left(e(\rho) \cdot \operatorname{ch}^{\omega}\left(\left(\bigwedge^{*} \mathfrak{n}_{-}\right) \otimes_{C} L(\Lambda)\right)\right)=(-1)^{\ell(w)-\hat{\ell}(w)}\left(e(\rho) \cdot \operatorname{ch}^{\omega}\left(\left(\bigwedge^{*} \mathfrak{n}_{-}\right) \otimes_{\boldsymbol{C}} L(\Lambda)\right)\right)$.

PROOF. It follows from Lemma 3.1.3 that

$$
\operatorname{ch}^{\omega}\left(\left(\bigwedge^{*} \mathfrak{n}_{-}\right) \otimes_{C} L(\Lambda)\right)=\operatorname{ch}^{\omega}\left(\bigwedge^{*} \mathfrak{n}_{-}\right) \cdot \operatorname{ch}^{\omega}(L(\Lambda))
$$

where

$$
\operatorname{ch}^{\omega}\left(\bigwedge^{*} \mathfrak{n}_{-}\right):=\operatorname{Tr}_{\bigwedge^{*} \mathfrak{n}_{-}} \omega^{-1} \exp
$$

Furthermore, we know from (2.2.7) that for $w \in \tilde{W}$,

$$
w\left(\operatorname{ch}^{\omega}(L(\Lambda))\right)=\operatorname{ch}^{\omega}(L(\Lambda)) .
$$

Hence we may assume that $\Lambda=0$.

It is well-known that $(-1)^{\ell\left(w w^{\prime}\right)}=(-1)^{\ell(w)}(-1)^{\ell\left(w^{\prime}\right)}$ for $w, w^{\prime} \in W$, and $(-1)^{\hat{\ell}\left(w w^{\prime}\right)}=$ $(-1)^{\hat{\ell}(w)}(-1)^{\hat{\ell}\left(w^{\prime}\right)}$ for $w, w^{\prime} \in \tilde{W}$. Thus, we may assume that $\hat{\ell}(w)=1$, i.e., $w=w_{i}$ for some $i \in \breve{I}$.

We set

$$
\Delta_{i}:=\Delta_{+} \cap\left(\sum_{k=0}^{N_{i}-1} \boldsymbol{Z} \alpha_{\omega^{k}(i)}\right), \quad \Delta(i):=\Delta_{+} \backslash \Delta_{i},
$$

and correspondingly

$$
\mathfrak{n}_{i}:=\bigoplus_{\alpha \in \Delta_{i}} \mathfrak{g}_{-\alpha}, \quad \mathfrak{n}(i):=\bigoplus_{\alpha \in \Delta(i)} \mathfrak{g}_{-\alpha}
$$

When $\sum_{k=0}^{N_{i}-1} a_{i, \omega^{k}(i)}=2$, we have

$$
\Delta_{i}=\left\{\alpha_{\omega^{k}(i)}\right\}_{k=0}^{N_{i}-1} .
$$

We call this case "Case (a)". When $\sum_{k=0}^{N_{i}-1} a_{i, \omega^{k}(i)}=1$, we have

$$
\Delta_{i}=\left\{\alpha_{\omega^{k}(i)}\right\}_{k=0}^{N_{i}-1} \sqcup\left\{\alpha_{\omega^{k}(i)}+\alpha_{\omega^{\left(N_{i} / 2\right)+k}(i)}\right\}_{k=0}^{N_{i} / 2-1} .
$$

We call this case "Case (b)". Since $\mathfrak{n}_{-}=\mathfrak{n}_{i} \oplus \mathfrak{n}(i)$, we have an isomorphism of $\mathfrak{h}$-modules:

$$
\bigwedge^{*} \mathfrak{n}_{-} \cong\left(\bigwedge^{*} \mathfrak{n}_{i}\right) \otimes_{C}\left(\bigwedge^{*} \mathfrak{n}(i)\right)
$$

Here we note that $\omega^{*}\left(\Delta_{i}\right)=\Delta_{i}$ and $\omega^{*}(\Delta(i))=\Delta(i)$. Then, since $\omega^{-1}\left(\mathfrak{g}_{\alpha}\right)=\mathfrak{g}_{\omega^{*}(\alpha)}$ for $\alpha \in \Delta$, we get that

$$
\left(\bigwedge^{*} \omega^{-1}\right)\left(\bigwedge^{*} \mathfrak{n}_{i}\right)=\bigwedge^{*} \mathfrak{n}_{i}
$$




$$
\left(\bigwedge^{*} \omega^{-1}\right)\left(\bigwedge^{*} \mathfrak{n}(i)\right)=\bigwedge^{*} \mathfrak{n}(i)
$$

Therefore, we can apply Lemma 3.1.3 to the $\mathfrak{h}$-module $\wedge^{*} \mathfrak{n}_{-} \cong\left(\bigwedge^{*} \mathfrak{n}_{i}\right) \otimes_{C}\left(\bigwedge^{*} \mathfrak{n}(i)\right)$ to obtain that

$$
\operatorname{ch}^{\omega}\left(\bigwedge^{*} \mathfrak{n}_{-}\right)=\operatorname{ch}^{\omega}\left(\bigwedge^{*} \mathfrak{n}_{i}\right) \cdot \operatorname{ch}^{\omega}\left(\bigwedge^{*} \mathfrak{n}(i)\right)
$$

where $\operatorname{ch}^{\omega}\left(\bigwedge^{*} \mathfrak{n}_{i}\right)$ and $\operatorname{ch}^{\omega}\left(\bigwedge^{*} \mathfrak{n}(i)\right)$ are defined by

$$
\begin{aligned}
& \operatorname{ch}^{\omega}\left(\bigwedge^{*} \mathfrak{n}_{i}\right):=\operatorname{Tr}_{\Lambda^{*} \mathfrak{n}_{i}} \bigwedge^{*} \omega^{-1} \exp , \\
& \operatorname{ch}^{\omega}\left(\bigwedge^{*} \mathfrak{n}(i)\right):=\operatorname{Tr}_{\bigwedge^{*} \mathfrak{n}(i)} \bigwedge^{*} \omega^{-1} \exp .
\end{aligned}
$$

STEP 1. First, we show that

$$
w_{i}\left(e(\rho) \cdot \operatorname{ch}^{\omega}\left(\bigwedge^{*} \mathfrak{n}_{i}\right)\right)=(-1)^{\ell\left(w_{i}\right)-\hat{\ell}\left(w_{i}\right)}\left(e(\rho) \cdot \operatorname{ch}^{\omega}\left(\bigwedge^{*} \mathfrak{n}_{i}\right)\right)
$$

To show this, as an ordered basis of $\mathfrak{n}_{i}$ we take $\left\{f_{\omega^{k}(i)}\right\}_{k=0}^{N_{i}-1}$ in Case (a), and $\left\{f_{\omega^{k}(i)}\right\}_{k=0}^{N_{i}-1} \sqcup$ $\left\{\dot{f}_{\omega^{k}(i)}\right\}_{k=0}^{N_{i} / 2-1}$ with $\dot{f}_{\omega^{k}(i)}:=\left[f_{\omega^{k}(i)}, f_{\omega^{\left(N_{i} / 2\right)+k}(i)}\right]$ for $0 \leq k \leq\left(N_{i} / 2\right)-1$ in Case (b).

Case (a): We can easily deduce that the only basis vectors of $\wedge^{*} \mathfrak{n}_{i}$ which make a contribution to the trace of $\wedge^{*} \omega^{-1}$ are the following two vectors:

$$
1 \in \bigwedge^{0} \mathfrak{n}_{i}=C \quad \text { and } \quad f_{i} \wedge f_{\omega(i)} \wedge \cdots \wedge f_{\omega^{N_{i}-1}(i)} .
$$

Hence we immediately obtain that

$$
\operatorname{ch}^{\omega}\left(\bigwedge^{*} \mathfrak{n}_{i}\right)=1+(-1)^{N_{i}-1} e\left(-\beta_{i}\right)
$$

where $\beta_{i}=\sum_{k=0}^{N_{i}-1} \alpha_{\omega^{k}(i)}$. Now we use Equation (2.2.5) to see that $w_{i}(\rho)=\rho-\beta_{i}$. So we have

$$
\begin{aligned}
w_{i}\left(e(\rho) \cdot \operatorname{ch}^{\omega}\left(\bigwedge^{*} \mathfrak{n}_{i}\right)\right) & =w_{i}\left(e(\rho) \cdot\left(1+(-1)^{N_{i}-1} e\left(-\beta_{i}\right)\right)\right) \\
& =e\left(\rho-\beta_{i}\right) \cdot\left(1+(-1)^{N_{i}-1} e\left(\beta_{i}\right)\right) \\
& =(-1)^{N_{i}-1} e(\rho) \cdot\left(1+(-1)^{N_{i}-1} e\left(-\beta_{i}\right)\right) \\
& =(-1)^{N_{i}-1} e(\rho) \cdot \operatorname{ch}^{\omega}\left(\bigwedge^{*} \mathfrak{n}_{i}\right) \\
& =(-1)^{\ell\left(w_{i}\right)-\hat{\ell}\left(w_{i}\right)}\left(e(\rho) \cdot \operatorname{ch}^{\omega}\left(\bigwedge^{*} \mathfrak{n}_{i}\right)\right),
\end{aligned}
$$

since it is seen from the form (2.2.3) of $w_{i}$ that $w_{i}\left(\beta_{i}\right)=-\beta_{i}$ and $\ell\left(w_{i}\right)=N_{i}$. 
Case (b): We can easily deduce that the only basis vectors of $\wedge^{*} \mathfrak{n}_{i}$ which make a contribution to the trace of $\wedge^{*} \omega^{-1}$ are the following four vectors:

$$
1 \in \bigwedge^{0} \mathfrak{n}_{i}=\boldsymbol{C}, f_{i} \wedge f_{\omega(i)} \wedge \cdots \wedge f_{\omega^{N_{i}-1}(i)}, \dot{f}_{i} \wedge \dot{f}_{\omega(i)} \wedge \cdots \wedge \dot{f}_{\omega^{\left(N_{i} / 2\right)-1}(i)},
$$

and

$$
f_{i} \wedge \cdots \wedge f_{\omega^{N_{i}-1}(i)} \wedge \dot{f}_{i} \wedge \cdots \wedge \dot{f}_{\omega^{\left(N_{i} / 2\right)-1}(i)} .
$$

Hence we immediately obtain that

$$
\begin{aligned}
\operatorname{ch}^{\omega}\left(\bigwedge^{*} \mathfrak{n}_{i}\right) & =\left(1+(-1)^{N_{i}-1} e\left(-\beta_{i}\right)\right)\left(1+(-1)(-1)^{\left(N_{i} / 2\right)-1} e\left(-\beta_{i}\right)\right) \\
& =\left(1+(-1)^{N_{i}-1} e\left(-\beta_{i}\right)\right)\left(1+(-1)^{N_{i} / 2} e\left(-\beta_{i}\right)\right)
\end{aligned}
$$

where $\beta_{i}=\sum_{k=0}^{N_{i}-1} \alpha_{\omega^{k}(i)}$. Now we use Equation (2.2.5) to see that $w_{i}(\rho)=\rho-2 \beta_{i}$. So we have

$$
\begin{aligned}
w_{i}\left(e(\rho) \cdot \operatorname{ch}^{\omega}\left(\bigwedge^{*} \mathfrak{n}_{i}\right)\right) \\
\quad=w_{i}\left(e(\rho) \cdot\left(1+(-1)^{N_{i}-1} e\left(-\beta_{i}\right)\right)\left(1+(-1)^{N_{i} / 2} e\left(-\beta_{i}\right)\right)\right) \\
=e\left(\rho-2 \beta_{i}\right) \cdot\left(1+(-1)^{N_{i}-1} e\left(\beta_{i}\right)\right)\left(1+(-1)^{N_{i} / 2} e\left(\beta_{i}\right)\right) \\
=e(\rho) \cdot(-1)^{(3 / 2) N_{i}-1}\left(1+(-1)^{N_{i}-1} e\left(-\beta_{i}\right)\right)\left(1+(-1)^{N_{i} / 2} e\left(-\beta_{i}\right)\right) \\
=(-1)^{(3 / 2) N_{i}-1} e(\rho) \cdot \operatorname{ch}^{\omega}\left(\bigwedge^{*} \mathfrak{n}_{i}\right) \\
\quad=(-1)^{\ell\left(w_{i}\right)-\hat{\ell}\left(w_{i}\right)}\left(e(\rho) \cdot \operatorname{ch}^{\omega}\left(\bigwedge^{*} \mathfrak{n}_{i}\right)\right),
\end{aligned}
$$

since it is seen from the form (2.2.3) of $w_{i}$ that $w_{i}\left(\beta_{i}\right)=-\beta_{i}$ and $\ell\left(w_{i}\right)=3 \cdot\left(N_{i} / 2\right)=$ $(3 / 2) N_{i}$.

STEP 2. Second, we show that

$$
w_{i}\left(\operatorname{ch}^{\omega}\left(\bigwedge^{*} \mathfrak{n}(i)\right)\right)=\operatorname{ch}^{\omega}\left(\bigwedge^{*} \mathfrak{n}(i)\right)
$$

which completes the proof of the proposition. Notice that $a_{\omega^{k}(i), \omega^{k}(i)}=2$ for all $0 \leq k \leq$ $N_{i}-1$, since $i \in \breve{I}$ implies $\sum_{k=0}^{N_{i}-1} a_{i, \omega^{k}(i)}>0$ by definition. Hence, the operators ad $e_{\omega^{k}(i)}$ and ad $f_{\omega^{k}(i)}$ are locally nilpotent on $\mathfrak{g}$ for all $0 \leq k \leq N_{i}-1$. We define linear automorphisms of $\mathfrak{g}$ by

$$
x_{k}^{\mathrm{ad}}:=\left(\exp \left(\operatorname{ad} f_{\omega^{k}(i)}\right)\right)\left(\exp \left(-\operatorname{ad} e_{\omega^{k}(i)}\right)\right)\left(\exp \left(\operatorname{ad} f_{\omega^{k}(i)}\right)\right)
$$


for $0 \leq k \leq N_{i}-1$, and then by

$$
X_{i}^{\mathrm{ad}}:= \begin{cases}\prod_{k=0}^{N_{i}-1} x_{k}^{\mathrm{ad}} & \text { in Case (a), } \\ \prod_{k=0}^{N_{i} / 2-1}\left(x_{k}^{\mathrm{ad}} x_{\left(N_{i} / 2\right)+k}^{\mathrm{ad}} x_{k}^{\mathrm{ad}}\right) & \text { in Case (b). }\end{cases}
$$

By [K, Lemma 3.8] we see that $X_{i}^{\text {ad }}\left(\mathfrak{g}_{\alpha}\right)=\mathfrak{g}_{w_{i}(\alpha)}$ for $\alpha \in \Delta$. Moreover, we immediately see that

$$
\omega^{-1}\left(X_{i}^{\mathrm{ad}}(v)\right)=\left(\omega^{-1} X_{i}^{\mathrm{ad}}\right)\left(\omega^{-1}(v)\right)
$$

for $v \in \mathfrak{g}$, where the linear automorphism $\omega^{-1} X_{i}^{\text {ad }}$ of $\mathfrak{g}$ is defined by

$$
\omega^{-1} X_{i}^{\mathrm{ad}}:= \begin{cases}\prod_{k=0}^{N_{i}-1} x_{k-1}^{\mathrm{ad}} & \text { in Case (a), } \\ \prod_{k=0}^{N_{i} / 2-1}\left(x_{k-1}^{\mathrm{ad}} x_{\left(N_{i} / 2\right)+k-1}^{\mathrm{ad}} x_{k-1}^{\mathrm{ad}}\right) & \text { in Case (b) },\end{cases}
$$

with $x_{-1}^{\text {ad }}:=x_{N_{i}-1}^{\text {ad }}$. We see easily that $\omega^{-1} X_{i}^{\text {ad }}=X_{i}^{\text {ad }}$, since, in Case (b),

$$
\begin{aligned}
x_{-1}^{\mathrm{ad}} x_{\left(N_{i} / 2\right)-1}^{\mathrm{ad}} x_{-1}^{\mathrm{ad}} & =x_{N_{i}-1}^{\mathrm{ad}} x_{\left(N_{i} / 2\right)-1}^{\mathrm{ad}} x_{N_{i}-1}^{\mathrm{ad}} \\
& =x_{\left(N_{i} / 2\right)-1}^{\mathrm{ad}} x_{N_{i}-1}^{\mathrm{ad}} x_{\left(N_{i} / 2\right)-1}^{\mathrm{ad}}
\end{aligned}
$$

(see, for example, [KP]). Thus we get the commutative diagram for $\alpha \in \Delta_{+}$:

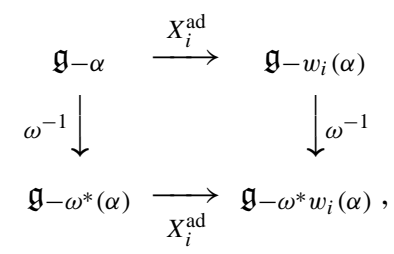

where $\omega^{*} w_{i}(\alpha)=w_{i} \omega^{*}(\alpha)$.

Because we see from Equation (2.2.5) that $w_{i}(\Delta(i))=\Delta(i)$, we deduce that

$$
X_{i}^{\mathrm{ad}}(\mathfrak{n}(i))=\mathfrak{n}(i) .
$$

By extending the linear automorphism $X_{i}^{\text {ad }}: \mathfrak{g} \rightarrow \mathfrak{g}$ to the linear automorphism

$$
\bigwedge^{*} X_{i}^{\mathrm{ad}}: \bigwedge^{*} \mathfrak{g} \rightarrow \bigwedge^{*} \mathfrak{g}
$$


in the usual way, we finally obtain the following commutative diagram from (3.2.19) and (3.2.20):

$$
\begin{array}{cc}
\Lambda^{*} \mathfrak{n}(i) \stackrel{\wedge^{*} X_{i}^{\mathrm{ad}}}{\longrightarrow} & \Lambda^{*} \mathfrak{n}(i) \\
\Lambda^{*} \omega^{-1} \downarrow & \downarrow^{*} \omega^{-1} \\
\Lambda^{*} \mathfrak{n}(i) \underset{\wedge^{*} X_{i}^{\mathrm{ad}}}{\longrightarrow} & \Lambda^{*} \mathfrak{n}(i) .
\end{array}
$$

Let $\chi \in\left(\mathfrak{h}^{*}\right)^{0}$ be a symmetric weight. Since $\omega^{*} w_{i}(\chi)=w_{i} \omega^{*}(\chi)=w_{i}(\chi)$, the following diagram commutes:

$$
\begin{aligned}
& \left(\wedge^{*} \mathfrak{n}(i)\right)_{\chi} \stackrel{\stackrel{\Lambda^{*} X_{i}^{\mathrm{ad}}}{\longrightarrow}}{\longrightarrow}\left(\Lambda^{*} \mathfrak{n}(i)\right)_{w_{i}(\chi)} \\
& \wedge^{*} \omega^{-1} \downarrow \\
& \left(\wedge^{*} \mathfrak{n}(i)\right)_{\chi} \underset{\wedge^{*} X_{i}^{\mathrm{ad}}}{\longrightarrow}\left(\Lambda^{*} \mathfrak{n}(i)\right)_{w_{i}(\chi)}
\end{aligned}
$$

It follows from this commutative diagram that for $\chi \in\left(\mathfrak{h}^{*}\right)^{0}$,

$$
\operatorname{Tr}\left(\left(\bigwedge^{*} \omega^{-1}\right) \mid\left(\bigwedge^{*} \mathfrak{n}(i)\right)_{\chi}\right)=\operatorname{Tr}\left(\left(\bigwedge^{*} \omega^{-1}\right) \mid\left(\bigwedge^{*} \mathfrak{n}(i)\right)_{w_{i}(\chi)}\right)
$$

Therefore, we conclude that

$$
w_{i}\left(\operatorname{ch}^{\omega}\left(\bigwedge^{*} \mathfrak{n}(i)\right)\right)=\operatorname{ch}^{\omega}\left(\bigwedge^{*} \mathfrak{n}(i)\right) .
$$

This proves the proposition.

COROLlaRY 3.2.3. Let $\Lambda \in P_{+} \cap\left(\mathfrak{h}^{*}\right)^{0}$ be a symmetric, dominant integral weight, and let $w \in \tilde{W}$ and $\beta \in \mathcal{S}(\Lambda) \cap\left(\mathfrak{h}^{*}\right)^{0}$. Then

$$
c_{(w, \beta)}=(-1)^{\ell(w)-\hat{\ell}(w)} \cdot(-1)^{\mathrm{ht}(\beta)-\widehat{\mathrm{ht}}(\beta)} .
$$

ProOf. First, we show that

$$
\operatorname{Tr}\left(\left.\Phi\right|_{\left(\left(\wedge^{*} \mathfrak{n}_{-}\right) \otimes_{C} L(\Lambda)\right)_{\Lambda-\beta}}\right)=(-1)^{\mathrm{ht}(\beta)-\widehat{\mathrm{ht}}(\beta)} .
$$

The element $\beta \in \mathcal{S}(\Lambda) \cap\left(\mathfrak{h}^{*}\right)^{0}$ can be written in the form $\beta=\sum_{k=1}^{l} \beta_{i_{k}}$, where $\beta_{i_{k}}=$ $\sum_{r=0}^{N_{i_{k}}-1} \alpha_{\omega^{r}\left(i_{k}\right)}$ for $1 \leq k \leq l$. Then obviously $\widehat{\operatorname{ht}}(\beta)=l$ and ht $(\beta)=\sum_{k=1}^{l} N_{i_{k}}$. Recall from (1.2.5) that we have

$$
\left(\left(\bigwedge^{*} \mathfrak{n}_{-}\right) \otimes_{C} L(\Lambda)\right)_{\Lambda-\beta}=\boldsymbol{C} v_{(1, \beta)}
$$


where $v_{(1, \beta)}=\hat{f}_{i_{1}} \wedge \hat{f}_{i_{2}} \wedge \cdots \wedge \hat{f}_{i_{l}} \otimes v_{\Lambda}$ with $\hat{f}_{i_{k}}:=f_{i_{k}} \wedge f_{\omega\left(i_{k}\right)} \wedge \cdots \wedge f_{\omega^{N_{i_{k}}-1}\left(i_{k}\right)}$ for $1 \leq k \leq l$. Then it follows that

$$
\begin{aligned}
\Phi\left(v_{(1, \beta)}\right) & =\left(\bigwedge^{*} \omega^{-1}\right)\left(\hat{f}_{i_{1}} \wedge \cdots \wedge \hat{f}_{i_{l}}\right) \otimes v_{\Lambda} \\
& =\left(\bigwedge^{*} \omega^{-1}\right)\left(\hat{f}_{i_{1}}\right) \wedge \cdots \wedge\left(\bigwedge^{*} \omega^{-1}\right)\left(\hat{f}_{i_{l}}\right) \otimes v_{\Lambda} .
\end{aligned}
$$

Because for each $1 \leq k \leq l$,

$$
\left(\bigwedge^{*} \omega^{-1}\right)\left(\hat{f}_{i_{k}}\right)=(-1)^{N_{i_{k}}-1} \hat{f}_{i_{k}}
$$

we deduce that

$$
\Phi\left(v_{(1, \beta)}\right)=(-1)^{\left(\sum_{k=1}^{l} N_{i_{k}}\right)-l} v_{(1, \beta)}=(-1)^{\mathrm{ht}(\beta)-\widehat{\mathrm{h}}(\beta)} v_{(1, \beta)} .
$$

Thus we have shown that

$$
\operatorname{Tr}\left(\left.\Phi\right|_{\left(\left(\wedge^{*} \mathfrak{n}_{-}\right) \otimes C L(\Lambda)\right)_{\Lambda-\beta}}\right)=(-1)^{\mathrm{ht}(\beta)-\widehat{\mathrm{ht}}(\beta)} .
$$

Now we set $j:=\ell(w)+\operatorname{ht}(\beta)$. Then the scalar

$$
\begin{aligned}
c_{(w, \beta)} & =\operatorname{Tr}\left(\left.\Phi_{j}\right|_{\left(\left(\wedge^{j} \mathfrak{n}_{-}\right) \otimes_{C} L(\Lambda)\right)_{(w, \beta) \circ \Lambda)}}\right) \\
& =\operatorname{Tr}\left(\left.\Phi\right|_{\left(\left(\wedge^{*} \mathfrak{n}_{-}\right) \otimes_{C} L(\Lambda)\right)_{(w, \beta) \circ \Lambda)}}\right)
\end{aligned}
$$

is the coefficient of $e(w(\Lambda+\rho-\beta))$ in $e(\rho) \cdot \operatorname{ch}^{\omega}\left(\left(\bigwedge^{*} \mathfrak{n}_{-}\right) \otimes_{C} L(\Lambda)\right)$, which is, by Proposition 3.2.2, equal to the coefficient of $e(\Lambda-\beta)$ in $\operatorname{ch}^{\omega}\left(\left(\Lambda^{*} \mathfrak{n}_{-}\right) \otimes_{C} L(\Lambda)\right)$ multiplied by $(-1)^{\ell(w)-\hat{\ell}(w)}$. But the coefficient of $e(\Lambda-\beta)$ in $\operatorname{ch}^{\omega}\left(\left(\bigwedge^{*} \mathfrak{n}_{-}\right) \otimes_{C} L(\Lambda)\right)$ is by definition $\operatorname{Tr}\left(\left.\Phi\right|_{\left(\left(\wedge^{*} \mathfrak{n}_{-}\right) \otimes_{C} L(\Lambda)\right)_{\Lambda-\beta}}\right)$. Thus, we obtain from (3.2.24) just proved that

$$
c_{(w, \beta)}=(-1)^{\ell(w)-\hat{\ell}(w)} \cdot(-1)^{\mathrm{ht}(\beta)-\widehat{\mathrm{ht}}(\beta)},
$$

as desired. This completes the proof.

Combining Proposition 3.2.1 with Corollary 3.2.3, we obtain our main result.

THEOREM 3.2.4. Let $\Lambda \in P_{+} \cap\left(\mathfrak{h}^{*}\right)^{0}$ be a symmetric, dominant integral weight, and let $j \geq 0$. Then

$$
\begin{aligned}
\operatorname{ch}^{\omega}\left(H_{j}\left(\mathfrak{n}_{-}, L(\Lambda)\right)\right)= & \sum_{\substack{w \in \tilde{W} \\
\beta \in \mathcal{S}(\Lambda) \cap\left(\mathfrak{h}^{*}\right)^{0} \\
\ell(w)+\mathrm{ht}(\beta)=j}}(-1)^{-(\ell(w)+\mathrm{ht}(\beta))} \cdot(-1)^{\hat{\ell}(w)+\widehat{\mathrm{ht}}(\beta)} e((w, \beta) \circ \Lambda) \\
= & \sum_{\substack{w \in \tilde{W} \\
\beta \in \mathcal{S}(\Lambda) \cap\left(\mathfrak{h}^{*}\right)^{0} \\
\ell(w)+\mathrm{ht}(\beta)=j}}(-1)^{\hat{\ell}(w)+\widehat{\mathrm{ht}}(\beta)-j} e((w, \beta) \circ \Lambda) .
\end{aligned}
$$

3.3. Application. As an application of Theorem 3.2.4, we give a new proof of the twining character formula (Theorem 2.2.1) for $L(\Lambda)$ of symmetric, dominant integral highest weight $\Lambda$. 
By virtue of Lemma 3.1.2, we can apply an Euler-Poincaré principle to deduce that

$$
\sum_{j \geq 0}(-1)^{j} \operatorname{ch}^{\omega}\left(H_{j}\left(\mathfrak{n}_{-}, L(\Lambda)\right)\right)=\sum_{j \geq 0}(-1)^{j} \operatorname{ch}^{\omega}\left(\left(\bigwedge^{j} \mathfrak{n}_{-}\right) \otimes_{\boldsymbol{C}} L(\Lambda)\right) .
$$

Also, by Lemma 3.1.2, we have for each $j \geq 0$,

$$
\operatorname{ch}^{\omega}\left(\left(\bigwedge^{j} \mathfrak{n}_{-}\right) \otimes_{C} L(\Lambda)\right)=\operatorname{ch}^{\omega}\left(\bigwedge^{j} \mathfrak{n}_{-}\right) \cdot \operatorname{ch}^{\omega}(L(\Lambda))
$$

Hence we get

$$
\begin{aligned}
\sum_{j \geq 0}(-1)^{j} \operatorname{ch}^{\omega}\left(\left(\bigwedge^{j} \mathfrak{n}_{-}\right) \otimes_{\boldsymbol{C}} L(\Lambda)\right) \\
=\sum_{j \geq 0}(-1)^{j} \operatorname{ch}^{\omega}\left(\bigwedge^{j} \mathfrak{n}_{-}\right) \cdot \operatorname{ch}^{\omega}(L(\Lambda)) \\
=\operatorname{ch}^{\omega}(L(\Lambda)) \cdot\left(\sum_{j \geq 0}(-1)^{j} \operatorname{ch}^{\omega}\left(\bigwedge^{j} \mathfrak{n}_{-}\right)\right) \\
=\operatorname{ch}^{\omega}(L(\Lambda)) \cdot\left(\sum_{j \geq 0}(-1)^{j} \operatorname{ch}^{\omega}\left(\left(\bigwedge^{j} \mathfrak{n}_{-}\right) \otimes_{C} L(0)\right)\right) \\
=\operatorname{ch}^{\omega}(L(\Lambda)) \cdot\left(\sum_{j \geq 0}(-1)^{j} \operatorname{ch}^{\omega}\left(H_{j}\left(\mathfrak{n}_{-}, L(0)\right)\right)\right),
\end{aligned}
$$

since $L(0)=C$ and $\operatorname{ch}^{\omega}(L(0))=e(0)=1$. Here, in the last equality above, we have used an Euler-Poincaré principle again.

On the other hand, we obtain from Theorem 3.2.4 that

$$
\sum_{j \geq 0}(-1)^{j} \operatorname{ch}^{\omega}\left(H_{j}\left(\mathfrak{n}_{-}, L(\Lambda)\right)\right)=\sum_{\substack{w \in \tilde{W} \\ \beta \in \mathcal{S}(\Lambda) \cap\left(\mathfrak{h}^{*}\right)^{0}}}(-1)^{\hat{\ell}(w)+\widehat{\mathrm{ht}}(\beta)} e((w, \beta) \circ \Lambda) .
$$

Putting all the above together, we conclude that

$$
\begin{aligned}
& \sum_{\substack{w \in \tilde{W} \\
\beta \in \mathcal{S}(\Lambda) \cap\left(\mathfrak{h}^{*}\right)^{0}}}(-1)^{\hat{\ell}(w)+\widehat{\mathrm{ht}}(\beta)} e((w, \beta) \circ \Lambda) \\
& \quad=\operatorname{ch}^{\omega}(L(\Lambda)) \cdot\left(\sum_{\substack{w \in \tilde{W} \\
\beta \in \mathcal{S}(0) \cap\left(\mathfrak{h}^{*}\right)^{0}}}(-1)^{\hat{\ell}(w)+\widehat{\mathrm{ht}}(\beta)} e((w, \beta) \circ 0)\right) .
\end{aligned}
$$

Thus, we have given a new proof of Theorem 2.2.1. 


\section{REFERENCES}

[B] R. E. Borcherds, Generalized Kac-Moody algebras, J. Algebra 115 (1988), 501-512.

[FRS] J. Fuchs, U. RAY AND C. SChWEIGERT, Some automorphisms of generalized Kac-Moody algebras, J. Algebra 191 (1997), 518-540.

[FSS] J. Fuchs, B. SChellekens AND C. Schweigert, From Dynkin diagram symmetries to fixed point structures, Comm. Math. Phys. 180 (1996), 39-97.

[GL] H. GARLAND AND J. LEPOWSKY, Lie algebra homology and the Macdonald-Kac formulas, Invent. Math. 34 (1976), 37-76.

[K] V. G. KAC, Infinite Dimensional Lie Algebras (3rd edition), Cambridge Univ. Press, Cambridge, 1990.

[KP] V. G. KAC AND D. H. Peterson, Defining relations of certain infinite-dimensional groups, Astérisque Numéro Hors Série (1985), 165-208.

[KK] S.-J. KANG AND J.-H. KWON, Graded Lie superalgebras, supertrace formula, and orbit Lie superalgebras, Proc. London Math. Soc. 81 (2000), 675-724.

[N1] S. NAITO, Kostant's formula for a certain class of generalized Kac-Moody algebras, Tôhoku Math. J. 44 (1992), 567-580.

[N2] S. NAITO, The strong Bernstein-Gelfand-Gelfand resolution for generalized Kac-Moody algebras. I-The existence of the resolution-, Publ. Res. Inst. Math. Sci. 29 (1993), 709-730.

[N3] S. NAITO, The strong Bernstein-Gelfand-Gelfand resolution for generalized Kac-Moody algebras. II-An explicit construction of the resolution, J. Algebra 167 (1994), 778-802.

[N4] S. NAITO, Twining character formula of Kac-Wakimoto type for affine Lie algebras, Represent. Theory 6 (2002), 70-100

INSTITUTE OF MATHEMATICS

UNIVERSITY OF TSUKUBA

TSUKUBA, IBARAKI 305-8571

JAPAN

E-mail address: naito@math.tsukuba.ac.jp 